\title{
ATTRACTORS IN INHOMOGENEOUS CONSERVATION LAWS AND PARABOLIC REGULARIZATIONS
}

\author{
HAITAO FAN AND JACK K. HALE
}

\begin{abstract}
The asymptotic behavior of inhomogeneous conservation laws is considered. The attractor of the equation is characterized. The relationship between attractors of the equation and that of its parabolic regularization is studied.
\end{abstract}

\section{INTRODUCTION}

In this paper, we shall characterize the global attractor for the hyperbolic equation

$$
u_{t}+f(u)_{x}=g(u), \quad t>0, \quad x \in S^{1},
$$

and study the relationships between the attractor of $(1.1)$ and that of the singularly perturbed parabolic equation

$$
u_{t}+f(u)_{x}=g(u)+\epsilon u_{x x},
$$

where $\epsilon>0$ is a small parameter. We assume that the functions $f, g$ satisfy the following hypotheses:

$\left(\mathrm{H}_{1}\right) \quad f \in \mathbb{C}^{2}(\mathbb{R}, \mathbb{R}), f^{\prime \prime}(u)>f_{0}>0$.

$\left(\mathrm{H}_{2}\right) \quad g(u)$ has finitely many zeroes, $a_{1}<a_{2}<\cdots<a_{2 k+1}$, all simple; and there is a constant $M_{0}>0$ such that

$$
u g(u)<0 \quad \text { for }|u|>M_{0} .
$$

We consider equations $(1.1)$ and (1.2) on the unit circle $S^{1}$, which is equivalent to imposing periodic boundary conditions of some period $L$. For (1.1), the initial data $u_{0}$ is assumed to be in the space

$$
B V\left(S^{1}\right)=\left\{u \in L^{1}\left(S^{1}\right): u_{x} \text { is a finite measure on } S^{1}\right\}
$$

of functions of bounded variation on $S^{1}$. Under the hypothesis $\left(\mathrm{H}_{1}\right)$, a solution $u(x, t)$ of $(1.1)$ is said to be an admissible solution if, for almost all $t>0$, the right- and left-hand limits of $u(x, t)$ with respect to $x$ exist and satisfy the entropy condition

$$
u\left(x^{-}, t\right) \geq u\left(x^{+}, t\right) .
$$

Received by the editors June 22, 1993.

1991 Mathematics Subject Classification. Primary 35L65, 35B40, 35B25, 34C35.

Key words and phrases. Attractors, conservation laws, dynamical systems.

Partially supported by NSF DMS-9005420 and DARPA 70NANB8H0860. 
It is known that the initial value problem has a unique weak admissible solution $u(x, t)$ with the property that $u \in C^{0}\left((0, \infty): L^{1}\left(S^{1}\right)\right), u(\cdot, t) \in$ $B V\left(S^{1}\right)$ (see, for example, Kruzkov (1970), Vol'pert (1967)). Throughout this paper, we consider only admissible solutions and, for any $u_{0} \in B V\left(S^{1}\right)$, we let $u^{0}\left(\cdot, t, u_{0}\right) \equiv \Phi^{0}(t) u_{0}$ be the solution of (1.1) with initial data $u_{0}$.

Since $B V\left(S^{1}\right) \subset L^{p}\left(S^{1}\right)$ for any $0<p<+\infty$, we can discuss the flow defined by (1.1) in $L^{p}\left(S^{1}\right) \cap B V\left(S^{1}\right)$. The positive orbit $\gamma^{+}(B)$ of a subset $B$ of $B V\left(S^{1}\right)$ is defined to be $\gamma^{+}(B)=\bigcup_{t \geq 0} \bigcup_{\varphi \in B} \Phi^{0}(t) \varphi$. The $\omega$-limit set $\omega(B)$ of $B$ in $L^{p}\left(S^{1}\right)$ is defined as $\omega_{L^{p}}(B)=\bigcap_{\tau \geq 0} C_{L^{p}} \bigcup_{t \geq \tau} \Phi^{0}(t) B$. If a solution is defined for all $t \leq 0$, we can define in a similar way the $\alpha$-limit set $\alpha_{L^{p}}$ by taking $t \rightarrow-\infty$. A set $M$ in $B V\left(S^{1}\right)$ is invariant if $\Phi^{0}(t) M=M$ for $t \geq 0$. A set $M$ in $B V\left(S^{1}\right)$ attracts a set $N$ in $B V\left(S^{1}\right)$ if $\operatorname{dist}_{L^{p}}\left(\Phi^{0}(t) N, M\right) \rightarrow 0$ as $t \rightarrow \infty$. If the closure in $L^{p}\left(S^{1}\right)$ of $\gamma^{+}(B)$ is compact, then $\omega_{L^{p}}(B)$ exists, is compact, invariant, and attracts $B$. Furthermore, if $B$ is connected, then $\omega_{L^{p}}(B)$ is connected. A set $\mathscr{A} \in B V\left(S^{1}\right)$ is said to be a global attractor if $\mathscr{A}$ is invariant and attracts arbitrarily bounded sets of $B V\left(S^{1}\right)$. The set $\mathscr{B}$ of globally defined and bounded orbits of $\Phi^{0}(t)$ consists of all $\varphi \in B V\left(S^{1}\right)$ such that $\Phi^{0}(t) \varphi$ is defined for all $t \in \mathbb{R}$ and $\bigcup_{t \in \mathbb{R}} \Phi^{0}(t) \varphi$ is bounded. If the global attractor exists, then $\mathscr{A}=\mathscr{B}$. For a further discussion of attractors, see, for example, Babin and Vishik (1989), Hale (1988), and Temam (1988).

We say that $\psi: \mathbb{R} \rightarrow \mathbb{R}$ is a traveling wave solution of (1.1) with wave speed $c$ if $\psi(x-c t)$ is a solution of $(1.1)$ for all $(x, t) \in S^{1} \times \mathbb{R}$. We say that $\omega_{L^{p}}\left(u_{0}\right)$ is a traveling wave with wave speed $c$ of $(1.1)$ if there is a real constant $c$ and a traveling wave solution $\psi: \mathbb{R} \rightarrow \mathbb{R}$ of (1.1) such that for any $\varphi \in \omega_{L^{p}}\left(u_{0}\right)$, $u^{0}(x, t, \varphi)=\psi(x-c t)$.

The following result has been proved by Fan and Hale (1992) and Lyberopoulos (1992).

Theorem 1.1. If $\left(\mathrm{H}_{1}\right),\left(\mathrm{H}_{2}\right)$ are satisfied and $u(x, t)$ is an admissible solution of $(1.1)$, then, for any $0<p<+\infty, \omega_{L^{p}}(u(\cdot, 0))$ exists and is either one of the zeros of $g$ or a traveling wave with wave speed $f^{\prime}\left(a_{2 m}\right)$, where $m=m\left(u_{0}\right)$ depends upon $u(\cdot, 0) \equiv u_{0}$.

In Section 3, we prove the following result concerning the globally defined and bounded solutions of (1.1).

Theorem 1.2. Let $\mathscr{A}_{0}$ be the set of $\varphi \in B V\left(S^{1}\right)$ such that the solution $\Phi^{0}(t) \varphi$ of (1.1) is defined and bounded for $t \in \mathbb{R}$. Then, for any $0<p<+\infty$, and any $\varphi \in \mathscr{A}_{0}, \alpha_{L^{p}}(\varphi)$ and $\omega_{L^{p}}(\varphi)$ exist and are either an equilibrium point or a traveling wave. Furthermore, $\mathscr{A}_{0}$ is a global attractor in $L^{p}\left(S^{1}\right)$; that is, for any bounded set $B \subset B V\left(S^{1}\right)$,

$$
\operatorname{dist}_{L^{p}\left(S^{1}\right)}\left(\Phi^{0}(t) B, \mathscr{A}_{0}\right) \rightarrow 0 \quad \text { as } t \rightarrow \infty .
$$

In Theorem 3.7, we give a more complete characterization of relationships between the $\alpha$-limit set and $\omega$-limit set of an orbit.

Section 4 is devoted to a discussion of some of the relationships between the solutions of (1.1) and (1.2). The space of initial data for (1.2) is taken to be the set of functions in $H^{2}\left(S^{1}\right)$. For any $u_{0} \in H^{2}\left(S^{1}\right)$, we let $u^{\epsilon}\left(\cdot, t, u_{0}\right) \equiv \Phi^{\epsilon}(t) u_{0}$ denote the solution of (1.2) with initial data $u_{0}$. The mapping $\Phi^{\epsilon}(t), t \geq 0$, is a $C^{0}$-semigroup on $H^{2}\left(S^{1}\right)$. 
As we have done above, for the semigroup $\Phi^{\epsilon}(t)$ on $H^{2}\left(S^{1}\right)$, we can define the positive orbit $\gamma^{+}(B)$ of a subset $B$ of $H^{2}\left(S^{1}\right)$, the $\omega$-limit set $\omega_{H^{2}}(B)$ of $B$, invariant sets and the global attractor for the semigroup $\Phi^{\epsilon}(t)$. It is known that the global attractor $\mathscr{A}_{\epsilon}$ exists for (1.2). Furthermore, for any $\varphi \in \mathscr{A}_{\epsilon}$, either $\omega_{H^{2}}(\varphi)$ is a traveling wave or $\alpha_{H^{2}}(\varphi)$ and $\omega_{H^{2}}(\varphi)$ belongs to the set of equilibrium points of (1.2) (see Fiedler and Mallet-Paret (1989)).

The ultimate goal would be to prove that the limit of $\mathscr{A}_{\epsilon}$ as $\epsilon \rightarrow 0$ is equal to $\mathscr{A}_{0}$. At this time, only partial results have been achieved. We proved that any orbit of (1.2) in $\mathscr{A}_{\epsilon}$, together with its $\alpha$-limit set and $\omega$-limit set converge almost everwhere to a similar orbit of (1.1) as $\epsilon \rightarrow 0$. See Theorems 4.1 and 4.3 for a precise statement. This topic will be treated in the near future.

We remark that $\operatorname{dim} \mathscr{A}_{0}=+\infty$, whereas $\operatorname{dim} \mathscr{A}_{\epsilon}<+\infty$ for each $\epsilon>0$. Therefore, it is very interesting to know if $\mathscr{A}_{\epsilon} \rightarrow \mathscr{A}_{0}$ as $\epsilon \rightarrow 0$ in the Hausdorff sense. We also remark that the tools for the discussion of the flows on $\mathscr{A}_{0}$ and $\mathscr{A}_{\epsilon}$ seem to be completely different. For $\mathscr{A}_{0}$, we exploit the theory of generalized characteristics, whereas, for $\mathscr{A}_{\epsilon}$, the discussion relies heavily upon invariant manifold theory and the oscillation number of a function.

\section{Preliminaries}

A fundamental tool in our investigation is the notion of generalized characteristic of Dafermos (1977). A Lipschitzian curve $x=\xi(t)$, defined on an interval $[a, b]$, is called a characteristic curve associated with the solution $u(x, t)$ of (1.1) if, for almost all $t \in[a, b]$,

$$
\dot{\xi}(t) \in\left[f ^ { \prime } \left(u\left(\xi(t)^{+}, t\right), f^{\prime}\left(u\left(\xi(t)^{-}, t\right)\right] .\right.\right.
$$

From Filippov (1960), for any $(\bar{x}, \bar{t}) \in \mathbb{R} \times(0, \infty)$, there exists at least one backward characteristic $\xi(t, \bar{x}, \bar{t})$, defined on a maximal interval $(s, \bar{t}], s \geq$ 0 , such that $\xi(\bar{t}, \bar{x}, \bar{t})=\bar{x}$. The set of backward characteristics through $(\bar{x}, \bar{t})$ spans a funnel confined between a minimal and a maximal backward characteristic through $(\bar{x}, \bar{t})$. We denote the minimal and maximal backward characteristics respectively by $\xi_{-}(t, \bar{x}, \bar{t}), \xi_{+}(t, \bar{x}, \bar{t})$.

A characteristic $\xi(\cdot)$, defined on $[a, b]$, is called genuine if $u\left(\xi(t)^{-}, t\right)=$ $u\left(\xi(t)^{+}, t\right)$ for almost all $t \in[a, b]$.

We need the following three results of Dafermos (1977) on characteristics.

Lemma 2.1. If $\xi:[a, b] \rightarrow \mathbb{R}$ is a characteristic, then, for almost all $t \in[a, b]$, we have

$$
\begin{aligned}
& \dot{\xi}(t)=f^{\prime}\left(u\left(\xi(t)^{ \pm}, t\right)\right) \quad \text { if } u\left(\xi(t)^{-}, t\right)=u\left(\xi(t)^{+}, t\right), \\
& \dot{\xi}(t)=\frac{f\left(u\left(\xi(t)^{+}, t\right)\right)-f\left(u\left(\xi(t)^{-}, t\right)\right)}{u\left(\xi(t)^{+}, t\right)-u\left(\xi(t)^{-}, t\right)} \text { if } u\left(\xi(t)^{-}, t\right)>u\left(\xi(t)^{+}, t\right) .
\end{aligned}
$$

Lemma 2.2. If $\xi:[a, b] \rightarrow \mathbb{R}$ is a genuine characteristic, then there is a function $v:[a, b] \rightarrow \mathbb{R}$ such that $(\xi, v)$ satisfies

$$
\dot{\xi}(t)=f^{\prime}(v(t)), \quad \dot{v}(t)=g(v(t))
$$

and, for almost every $t \in(a, b)$,

$$
v(t)=u\left(\xi(t)^{+}, t\right)=u\left(\xi(t)^{-}, t\right) .
$$


Lemma 2.3. The minimal and maximal characteristics $\xi_{-}(t, \bar{x}, \bar{t}), \xi_{+}(t, \bar{x}, \bar{t})$ are genuine and are obtained by solving equations (2.3) with initial conditions at $t=\bar{t}$ respectively given by $\left(\bar{x}, u\left(\bar{x}^{-}, \bar{t}\right)\right)$ and $\left(\bar{x}, u\left(\bar{x}^{+}, \bar{t}\right)\right)$.

Corollary 2.4. Two distinct genuine characteristics can intersect only at their endpoints.

The following result can be found in Fan and Hale (1992) and is based on Kruzkov (1970).

Theorem 2.5. If $f \in C^{1}(\mathbb{R}, \mathbb{R})$ and $\left(\mathrm{H}_{2}\right)$ is satisfied, then the solution $u(x, t)$ of (1.1) satisfies the estimate

$$
\|u(\cdot, t)\|_{L^{\infty}\left(S^{1}\right)} \leq \max \left(\left\|u_{0}\right\|_{L^{\infty}\left(S^{1}\right)}, M_{0}\right), \quad \text { for } t \geq 0 .
$$

Corollary 2.6. Backward characteristics through any $(\bar{x}, \bar{t})$ are defined on $[0, \bar{t}]$.

\section{THE CONNECTING ORBITS AND ATTRACTOR OF (1.1)}

In this section, we study the composition of the attractor of (1.1) and some properties of connecting orbits.

We define the range $\mathscr{R}(u(\cdot, t))$ of a function $u(\cdot, t)$ by

$$
\mathscr{R}(u(\cdot, t)):=\{u(x \pm, t): x \in \mathbb{R}\} .
$$

In Fan and Hale (1992), we proved the following lemmas.

Lemma 3.1. If $u(x, t)$ is a solution of $(1.1)$, then $\mathscr{R}(u(\cdot, t))$ is a closed interval.

Lemma 3.2. There exists a constant $T_{0}$, depending only on the period $L$, the function $f$, and the zero set $\left\{a_{1}, \ldots, a_{2 k+1}\right\}$ of $g(u)$ such that, for $t>T_{0}$, $\mathscr{R}(u(\cdot, t))$ contains no more than one point of the set $\left\{a_{1}, \ldots, a_{2 k+1}\right\}$. Furthermore,

$$
T_{0}=\frac{L}{\min \left\{\left|f^{\prime}\left(a_{m}\right)-f^{\prime}\left(a_{n}\right)\right|: 1 \leq n, m \leq 2 k+1, m \neq n\right\}} .
$$

In the following result, we let $T V_{I}(u)$ be the total variation of a function $u$ on the interval $I$.

Lemma 3.3. For any $r>0$, there is a positive constant $C=C(r)$ such that, if $u(x, t)$ is a solution of (1.1) with $\|u(\cdot, 0)\|_{L^{\infty}}<r$, then, for $t \geq 0$,

$$
T V_{[0, L]}(u(\cdot, t)) \leq T V_{[0, L]}(u(\cdot, 0))+2 C L .
$$

Proof. We consider first the decreasing variation of the solution $u(x, t)$. Let $\xi(t)$ be an extremum backward characteristic and let $\xi(\bar{t})=y_{1} \leq y_{2} \leq \ldots \leq$ $y_{2 n}=\xi(\bar{t})+L$ be a partition with $u\left(y_{2 j-1}^{-}, \bar{t}\right)>u\left(y_{2 j}^{+}, \bar{t}\right), j=1, \ldots, n$.

For each $j=1,2, \ldots, n$, let $\xi_{-}\left(t ; y_{2 j-1}, \bar{t}\right) \equiv \xi_{2 j-1}(t) \quad\left(\right.$ resp. $\xi_{+}\left(t, y_{2 j}, \bar{t}\right)$ $\left.\equiv \xi_{2 j}(t)\right)$ be the minimal (resp. maximal) backward characteristic through the point $\left(y_{2 j-1}, \bar{t}\right)$ (resp. $\left.\left(y_{2 j-1}, \bar{t}\right)\right)$. From Lemma 2.2, the maximal backward characteristic $\xi_{2 j}$ satisfies the systems of equations:

$$
\begin{aligned}
\frac{d \xi_{2 j}}{d t} & =f^{\prime}\left(v_{2 j}(t)\right), \frac{d v_{2 j}}{d t}=g\left(v_{2 j}(t)\right), \\
\xi_{2 j}(\bar{t}) & =y_{2 j}, v_{2 j}(\bar{t})=u\left(y_{2 j}^{+}, \bar{t}\right), \\
v_{2 j}(t) & =u\left(\xi_{2 j}(t)^{+}, t\right)=u\left(\xi_{2 j}(t)^{-}, t\right) \quad \text { a.e. in }(0, \bar{t})
\end{aligned}
$$


and the minimal backward characteristic $\xi_{2 j-1}$ satisfies the systems of equations:

$$
\begin{aligned}
\frac{d \xi_{2 j-1}}{d t} & =f^{\prime}\left(v_{2 j-1}(t)\right), \frac{d v_{2 j-1}}{d t}=g\left(v_{2 j-1}(t)\right) \\
\xi_{2 j-1}(\bar{t}) & =y_{2 j-1}, v_{2 j-1}(\bar{t})=u\left(y_{2 j-1}^{-}, \bar{t}\right), \\
v_{2 j-1}(t) & =u\left(\xi_{2 j-1}(t)^{+}, t\right)=u\left(\xi_{2 j-1}(t)^{-}, t\right) \quad \text { a.e. in }(0, \bar{t})
\end{aligned}
$$

It is easily seen that

$$
\frac{d\left(v_{2 j-1}-v_{2 j}\right)}{d t}=g\left(v_{2 j-1}\right)-g\left(v_{2 j}\right)=G(t)\left(v_{2 j-1}-v_{2 j}\right) .
$$

Since $v_{2 j-1}(\bar{t})>v_{2 j}(\bar{t})$, uniqueness of the solutions of the initial value problem for ordinary differential equations implies that $v_{2 j-1}(t)>v_{2 j}(t)$ for $t \in[0, t]$. since $v_{2 j-1}(\bar{t})>v_{2 j}(\bar{t})$. This fact, together with (3.3), yields

$$
\frac{d\left(\xi_{2 j-1}-\xi_{2 j}\right)}{d t}=f^{\prime}\left(v_{2 j-1}\right)-f^{\prime}\left(v_{2 j}\right)=f^{\prime \prime}(\sigma)\left(v_{2 j-1}-v_{2 j}\right)>0,
$$

for $t \in(0, \bar{t})$. From equations (3.4), (3.5), we deduce that

$$
\frac{d\left(v_{2 j-1}-v_{2 j}\right)}{d t}=\frac{g\left(v_{2 j-1}\right)-g\left(v_{2 j}\right)}{f^{\prime}\left(v_{2 j-1}\right)-f^{\prime}\left(v_{2 j}\right)} \frac{d\left(\xi_{2 j-1}-\xi_{2 j}\right)}{d t}
$$

or

$$
\begin{aligned}
0< & v_{2 j-1}(\bar{t})-v_{2 j}(\bar{t})=v_{2 j-1}(0)-v_{2 j}(0) \\
& +\int_{0}^{\bar{t}} \frac{g\left(v_{2 j-1}\right)-g\left(v_{2 j}\right)}{f^{\prime}\left(v_{2 j-1}\right)-f^{\prime}\left(v_{2 j}\right)} \frac{d\left(\xi_{2 j-1}-\xi_{2 j}\right)}{d t} d t .
\end{aligned}
$$

Using this relation and (3.5), the $L^{\infty}$-boundedness of $u(x, t)$, and the facts that $u_{j}(x, 0) \in\left[u_{0}\left(\xi_{j}(0)^{+}, 0\right), u_{0}\left(\xi_{j}(0)^{-}, 0\right)\right]$ and there is a $\delta(r)>0$ such that $f^{\prime \prime}(u) \geq \delta(r)>0$ for $|u| \leq r$, we deduce that

$$
\begin{aligned}
\sum_{j=1}^{n}\left(u\left(y_{2 j-1}^{-}, \bar{t}\right)-u\left(y_{2 j}^{+}, \bar{t}\right)\right)=\sum_{j=1}^{n}\left(v_{2 j-1}(\bar{t})-v_{2 j}(\bar{t})\right) \\
\leq \sum_{j=1}^{n}\left(v_{2 j-1}(0)-v_{2 j}(0)\right)+\sum_{j=1}^{n} C \int_{0}^{\bar{t}} \frac{d\left(\xi_{2 j-1}-\xi_{2 j}\right)}{d t} d t \\
\leq T V^{-}\left\{u_{0}(x), x \in[0, L]\right\}+C \int_{0}^{\bar{t}} \frac{d\left(\xi_{2 j-1}-\xi_{2 j}\right)}{d t} d t \\
\leq T V^{-}\left\{u_{0}(x), x \in[0, L]\right\}+C L,
\end{aligned}
$$

where $T V^{-}$denotes the decreasing variation.

For the increasing variation of $u(x, t)$, we employ a similar argument using a partition with $u\left(y_{2 j-1}^{+}, \bar{t}\right)<u\left(y_{2 j}^{-}, \bar{t}\right), j=1, \ldots, n$, and the maximal and minimal characteristics $\xi_{+}\left(t ; y_{2 j-1}, \bar{t}\right), \xi_{-}\left(t, y_{2 j}, \bar{t}\right)$. This will complete the proof.

Lemma 3.4. For any $r>0$, there exists a positive constant $C=C(r)$ such that, if $u(x, t)$ is a solution of $(1.1)$ with $u(x, 0)=u_{0}(x)$ and $T V_{[0, L]}\left(u_{0}\right)+$ $\left\|u_{0}\right\|_{L^{\infty}}<r$, then, for any $\Delta t>0$,

$$
\int_{0}^{L}|u(x, t+\Delta t)-u(x, t)| d x \leq C|\Delta t| .
$$


Proof. Integrating (1.1) with respect to $t$, we obtain

$$
u(x, t+\Delta t)-u(x, t)=-\int_{t}^{t+\Delta t} f(u(x, s))_{x} d s+\int_{t}^{t+\Delta t} g(u(x, s)) d s .
$$

If we use Lemma 3.3, then

$\int_{0}^{L}|u(x, t+\Delta t)-u(x, t)| d x \leq\left|\int_{t}^{t+\Delta t} \int_{0}^{L}\left(\left|f^{\prime}(u)\right|\left|u_{x}\right|+|g(u)|\right) d x d t\right| \leq C|\Delta t|$,

which is (3.6).

Corollary 3.5. If $B \subset B V\left(S^{1}\right)$ and $\max _{u_{0} \in B}\left(T V\left\{u_{0}(x), x \in S^{1}\right\}\right)<+\infty$, then there is a positive constant $C=C(B)$ such that

(i) $\max _{u_{0} \in B} T V_{(x, t) \in S^{1} \times[0, T]}\left(\Phi^{0}(t) u_{0}\right)<C$ for any $T>0$;

(ii) the set of solutions $\Phi^{0}(t) B, t \in \mathbb{R}_{+}$, is compact in the sense of pointwise convergence;

(iii) the set of solutions $\Phi^{0}(t) B$ is compact in $L^{p}\left(S^{1} \times[0, T]\right)$ for any $T>0$ and any $p>0$.

Proof. We recall that the necessary and sufficient condition for a function to be of bounded total variation on the set $U=S^{1} \times[0, T], T>0$, is the existence of a positive constant $C$ such that

$$
\int_{U}|u(y+\Delta y)-u(y)| d y \leq C\|\Delta y\|_{\mathbb{R}^{2}} .
$$

Assertion (i) is now a direct consequence of Lemmas 3.3, 3.4.

Assertion (ii) is a consequence of Helly's Theorem.

Assertion (iii) is a consequence of. (ii) and the $L^{\infty}$-boundedness of $\Phi^{0}(t) B$ (Theorem 2.5).

Let $\mathscr{A}_{0}$ be the set of globally defined and bounded solutions of (1.1); that is,

$$
\mathscr{A}_{0}=\left\{u_{0} \in B V\left(S^{1}\right): \Phi^{0}(t) u_{0} \text { is defined and bounded for } t \in \mathbb{R}\right\} .
$$

Also, for any bounded set $B \subset B V\left(S^{1}\right)$, we define

$$
\omega_{L^{p}\left(S^{1}\right)}(B)=\bigcap_{\tau \geq 0} \mathrm{Cl}_{L^{p}\left(S^{1}\right)} \bigcup_{t \geq \tau} \Phi^{0}(t) B
$$

as the $\omega$-limit set of $B$ in $L^{p}\left(S^{1}\right)$.

We can prove the following result.

Theorem 3.6. For any bounded set $B$ in $B V\left(S^{1}\right)$, the $\omega$-limit set $\omega_{L^{p}\left(S^{1}\right)}(B)$ exists, is compact in $L^{p}\left(S^{1}\right)$, belongs to $B V\left(S^{1}\right)$, and is invariant under $\Phi^{0}(t)$. Furthermore, $\mathscr{A}_{0}$ is the global attractor of $(1.1)$ in $L^{p}\left(S^{1}\right)$; that is,

$$
\operatorname{dist}_{L^{p}\left(S^{1}\right)}\left(\Phi^{0}(t) B, \mathscr{A}_{0}\right) \rightarrow 0 \quad \text { as } t \rightarrow+\infty \text {. }
$$

Proof. The fact that $\omega_{L^{p}\left(S^{1}\right)}(B)$ exists, is compact in $L^{p}\left(S^{1}\right)$, and belongs to $B V\left(S^{1}\right)$ is clear from Lemma 3.3 and Corollary 3.5. The invariance under $\Phi^{0}(t)$ will be apparent from the proof we give of (3.9).

There is a constant $C$ such that $T V[0, L] u_{0}+\left\|u_{0}\right\|_{L^{\infty}}<C$ for all $u_{0} \in B$. If (3.9) is not satisfied, then there are an $\epsilon_{0}>0$ and sequences $\left\{u_{0 j}\right\} \subset B$ and $\left\{t_{j}\right\} \subset(0, \infty), t_{j} \rightarrow \infty$ monotonically as $j \rightarrow \infty$, such that

$$
\operatorname{dist}_{L^{p}\left(S^{\prime}\right)}\left(\Phi^{0}\left(t_{j}\right) u_{0 j}, \mathscr{A}_{0}\right) \geq \epsilon_{0}
$$


for $j=1,2, \ldots$. For the fixed integer $N>0$, let $j_{N}$ be so large that $t_{j}>N$ for all $j \geq j_{N}$. If we define $u_{j}(x, t)=\Phi^{0}\left(t_{j}+t\right) u_{0 j}$ for $j \geq j_{N}$ and $t \geq-N$, then we deduce from Lemma 3.3 and (3.4) that there is a constant $C_{N}$ such that $T V_{(x, t) \in S^{1} \times[-N, N]}\left(u_{j}\right) \leq C_{N}$. As a consequence, we can extract a subsequence of the $u_{j}$, which we denote with the same symbols, such that $u_{j}(x, t) \rightarrow u(x, t)$ almost everywhere in $S^{1} \times[-N, N]$. By the diagonalization process, we can find a subsequence, which we again denote by $u_{j}$ and a function $u(x, t)$ such that $u_{j}(x, t) \rightarrow u(x, t)$ almost everywhere in $S^{1} \times \mathbb{R}$ as $j \rightarrow \infty$. Furthermore, $T V_{(x ; t) \in S^{1} \times[-N, N]}\left(u_{j}\right) \leq C_{N}$ for every $N$. By Lemma 3.3, we may also assume, restricting the subsequence if necessary, that $u_{j}(x, 0) \rightarrow u(x, 0)$ almost everywhere in $S^{1}$ as $j \rightarrow \infty$. The function $u(x, t)$ is an admissible weak solution of (1.1) and hence $u(x, 0) \in \mathscr{A}_{0}$. This leads to a contradiction of (3.10) since pointwise convergence and $L^{\infty}$ - boundedness imply $L^{p}$-convergence. The theorem is proved.

Now we study the structure of solutions of (1.1) in the attractor. In Theorem 1.1 , we have noted that the $\omega$-limit set of any orbit of $(1.1)$ is either a constant $\in\left\{a_{1}, \ldots, a_{2 k+1}\right\}$ or a traveling wave with speed $f^{\prime}\left(a_{2 l}\right), l \in\{1,2, \ldots, k\}$. If $u_{c}(x, t)$ is a solution of (1.1) which exists for $t \in \mathbb{R}$ and $\varphi(x-c t)$ is a traveling wave such that $u_{c}(x, t)-\varphi(x-c t) \rightarrow 0$ as $t \rightarrow \infty$, then we say that $u_{c}(x, t)$ connects to $\varphi$ at $t=+\infty$. Similarly, if there exists a traveling wave $\psi(x-c t)$ such that $u_{c}(x, t)-\psi(x-c t) \rightarrow 0$ as $t \rightarrow-\infty$, then we say that $u_{c}(x, t)$ connects to $\psi$ at $t=-\infty$. For any function $\varphi$ on $[0, L]$, we define the oscillation number Card $\mathscr{N}_{\varphi}$ as the cardinal number of the set

$$
\mathscr{N}_{\varphi}=\left\{x \in[0, L]: \varphi\left(x^{+}\right)=\varphi\left(x^{-}\right)=0\right\} .
$$

\section{Theorem 3.7.}

(i) Any element of $\mathscr{A}_{0}$ must connect at $t=+\infty$ to either a traveling wave or a constant $\in\{u: g(u)=0\}$.

(ii) If $u_{c}(x, t)$ is not a traveling wave and connects to a nonconstant traveling wave $\varphi$ of (1.1) at $t=+\infty$ with speed $f^{\prime}\left(a_{2 m}\right)$ for some $m \in$ $\{1, \ldots, k\}$, then $u_{c}(x, t)$ must connect to either a traveling wave $\psi$ of (1.1) at $t=-\infty$ with the same speed or $\varphi$ must connect to a constant solution $\psi \equiv a_{2 m}$ at $t=-\infty$. Furthermore, Card $\mathscr{N}_{\varphi-a_{2 m}} \leq \mathscr{N}_{\psi-a_{2 m}}$.

(iii) If $u_{c}(x, t)$ connects to a constant $b$ at $t=+\infty$ and $u_{c}(x, t) \neq b$ for all $x, t$, then $b=a_{2 m+1}$ for some $m$ and $u_{c}(x, t)$ connects at $t=-\infty$ to a traveling wave $\psi$ with speed either $f^{\prime}\left(a_{2 m}\right)$ or $f^{\prime}\left(a_{2 m+2}\right)$.

Proof. Conclusion (i) is a restatement of Theorem 1.1.

We now prove (ii). Since $u_{c}(x, t)$ connects to $\varphi\left(x-f^{\prime}\left(a_{2 m}\right) t\right)$ at $+\infty$, from Lemma 3.5 of Hale and Fan (1992), $a_{2 m}$ is in the interior of $\mathscr{R}\left(\varphi\left(\cdot-f^{\prime}\left(a_{2 m}\right) t\right)\right)$. Therefore, we deduce from Lemma 2.3 that

$$
a_{2 m} \in R\left(u_{c}(\cdot, t)\right) \subset\left(a_{2 m-1}, a_{2 m+1}\right) \text { for } t \geq T_{0} .
$$

We claim that $(3.11)$ is true for any $t \in \mathbb{R}$. Indeed, let $(\bar{x}, T) \in S^{1} \times \mathbb{R}$ be such that either $u_{c}\left(\bar{x}^{+}, T\right)$ or $u_{c}\left(\bar{x}^{-}, T\right)=a_{2 m}$. Then $\xi(t ; \bar{x}, T)=$ $f^{\prime}\left(a_{2 m}\right)(t-T)+\bar{x}, t<T$, is an extremum backward characteristic of $(1.1)$ and $u_{c}\left(\xi(t ; \bar{x}, T)^{ \pm}, t\right)=a_{2 m}$ a.e. in $(-\infty, T)$. Hence, $a_{2 m} \in R\left(u_{c}(\cdot, t)\right)$ for $t \in \mathbb{R}$. This fact together with Lemma 3.2 imply that

$$
a_{2 m} \in R\left(u_{c}(\cdot, t)\right) \subset\left(a_{2 m-1}, a_{2 m+1}\right) \text { for } t \in \mathbb{R} \text {. }
$$


By translation of variables, we may assume, without loss of generality, that $f^{\prime}\left(a_{2 m}\right)=0$ and $u_{c}(0, T)=a_{2 m}=u_{c}(L, T)$ for some $T \in \mathbb{R}$. It follows that

$$
\xi_{1}(t)=0 \quad \text { and } \quad \xi_{2}(t)=L, \quad-\infty<t<T,
$$

are genuine backward characteristics of (1.1). We consider the extremal backward characteristcs $\xi_{ \pm}(t ; \bar{x}, \bar{t})$ of $(1.1)$ emanating from $(\bar{x}, \bar{t})$ for $\bar{x} \in(0, L)$, $\bar{t}<T$. For all $t<\bar{t}$, these functions satisfy

$$
\left\{\begin{array}{l}
\frac{d \xi_{ \pm}(t ; \bar{x}, \bar{t})}{d t}=f^{\prime}(v(t)), \quad \frac{d v(t)}{d t}=g(v(t)), \\
\xi_{ \pm}(\bar{t} ; \bar{x}, \bar{t})=\bar{x}, \quad v(\bar{t})=u_{c}\left(\bar{x}^{ \pm}, \bar{t}\right),
\end{array}\right.
$$

and, for almost all $t \leq \bar{t}, v(t)=u\left(\xi_{ \pm}(t, \bar{x}, \bar{t}), t\right)$. From Corollary 2.4 and (3.13), we have $\xi_{ \pm}(t ; \bar{x}, \bar{t}) \in(0, L)$ for $t<\bar{t}$. Since $f^{\prime}(u)>0$ on $(0, L)$, these last two remarks and (3.14) imply that $\xi_{ \pm}(t ; \bar{x}, \bar{t})$ is monotone with respect to $t$. Thus, the limits

$$
\lim _{t \rightarrow-\infty} \xi_{ \pm}(t ; \bar{x}, \bar{t})=\xi_{ \pm}(-\infty ; \bar{x}, \bar{t}) \in[0, L]
$$

exist for any $(\bar{x}, \bar{t}) \in[0, L] \times(-\infty, T)$.

We now investigate the limit of $\xi_{ \pm}(-\infty ; \bar{x}, \bar{t})$ as $\bar{t} \rightarrow-\infty$. We claim that either there is a $y(x)$ such that

$$
\lim _{\bar{t} \rightarrow-\infty} \xi_{ \pm}(-\infty ; \bar{x}, \bar{t})=y(\bar{x})
$$

or there exist $y_{1}(\bar{x})<\bar{x}<y_{2}(\bar{x})$ such that, for any sequence $t_{j} \rightarrow-\infty$, we have

$$
\lim _{t_{j} \rightarrow-\infty} \xi_{ \pm}\left(-\infty ; \bar{x}, t_{j}\right)=\text { either } y_{1}(\bar{x}) \text { or } y_{2}(\bar{x}) .
$$

To prove this claim, we let $\xi(-\infty ; \bar{x}, t)$ designate either $\xi_{+}(-\infty ; \bar{x}, t)$ or $\xi_{-}(-\infty ; \bar{x}, t)$. If (3.16) does not hold, then there are two sequences $t_{j} \rightarrow-\infty$, $\bar{t}_{j} \rightarrow-\infty$, such that

$$
\lim _{t_{j} \rightarrow-\infty} \xi\left(-\infty ; \bar{x}, t_{j}\right)=y_{1}(\bar{x}), \quad \lim _{\bar{t}_{j} \rightarrow-\infty} \xi\left(-\infty ; \bar{x}, \bar{t}_{j}\right)=y_{2}(\bar{x})>y_{1}(\bar{x}) .
$$

We first prove that $y_{2}(\bar{x})>\bar{x}>y_{1}(\bar{x})$. If the contrary holds, for example, if $\bar{x}>y_{2}(\bar{x})>y_{1}(\bar{x})$, then there are integers $j_{1}, j_{2}$ such that $t_{j_{1}}>\bar{t}_{j_{2}}$ such that $\bar{x}>\xi\left(-\infty, \bar{x}, t_{j_{1}}\right)>\xi\left(-\infty, \bar{x}, \bar{t}_{j_{2}}\right)$. These two inequalities imply that $\xi\left(t, \bar{x}, t_{j_{1}}\right)$ and $\xi\left(t, \bar{x}, \bar{t}_{j_{2}}\right)$ intersect, which contradicts Corollary 2.4. Thus, $y_{2}(\bar{x})>\bar{x}>y_{1}(\bar{x})$. For the same reason, there can be no other limit points of $\xi(-\infty, \bar{x}, \bar{t})$ as $\bar{t} \rightarrow-\infty$. This completes the proof of the claim and so either (3.16) or (3.17) holds.

Our next objective is to show that (3.16) holds for almost all points in $[0, L]$. If (3.17) holds, then there exist $N>0$ such that, for all $t<-N$, we have either

$$
\left|\xi_{-}(-\infty, \bar{x}, t)-y_{1}(\bar{x})\right|<\frac{1}{4}\left|y_{1}(\bar{x})-\bar{x}\right|
$$

or

$$
\left|\xi(-\infty, \bar{x}, t)-y_{2}(\bar{x})\right|<\frac{1}{4}\left|y_{2}(\bar{x})-\bar{x}\right|
$$


Let $t_{1}$ (resp. $t_{2}$ ) be a value of $t$ such that (3.18) (resp. (3.19)) is satisfied. For $t<\min \left\{t_{1}, t_{2}\right\}$ and $\xi(t, \bar{x}, \bar{t})$ denoting either $\xi_{+}(t, \bar{x}, \bar{t})$ or $\xi_{-}(t, \bar{x}, \bar{t})$, we define

(3.20a)

$S^{-}(t) \equiv\left\{x \in\left[\xi\left(t, \bar{x}, t_{1}\right), \xi\left(t, \bar{x}, t_{2}\right)\right]:\left|\xi_{-}(-\infty, \bar{x}, t)-y_{1}(\bar{x})\right|<\frac{1}{4}\left|y_{1}(\bar{x})-\bar{x}\right|\right\}$,

$S^{+}(t) \equiv\left\{x \in\left[\xi\left(t, \bar{x}, t_{1}\right), \xi\left(t, \bar{x}, t_{2}\right)\right]:\left|\xi_{+}(-\infty, \bar{x}, t)-y_{2}(\bar{x})\right|<\frac{1}{4}\left|y_{2}(\bar{x})-\bar{x}\right|\right\}$.

By Corollary 2.4, we see that $S^{ \pm}(t)$ are intervals and $S^{+}(t) \cup S^{-}(t)=$ $\left[\xi\left(t, \bar{x}, t_{1}\right), \xi\left(t, \bar{x}, t_{2}\right)\right]$. Thus, we can write

$$
S^{-}(t)=\left[\xi\left(t, \bar{x}, t_{1}\right), \chi(t)\right], \quad S^{+}(t)=\left[\chi(t), \xi\left(t, \bar{x}, t_{2}\right)\right] .
$$

Using a similar proof as the ones for Corollary 3.11 and Lemma 3.13 of Fan and Hale (1992), we observe that $\chi(t)$ is a characteristic curve and that $\chi(t) \rightarrow \bar{x}$ as $t \rightarrow-\infty$.

For any $x \in\left(y_{1}(\bar{x}), \bar{x}\right)$, we want to show that (3.16) holds. Let $\left\{t_{j}\right\}$ be such that $\lim _{t_{j} \rightarrow-\infty} \xi\left(-\infty, \bar{x}, t_{j}\right)=y_{1}(\bar{x})<\bar{x}$. For each $t_{j}$, there exists a positive constant $N_{j}$ such that $\xi\left(t, \bar{x}, t_{j}\right)<x$ for $t<-N_{j}$. Thus, $\xi\left(t, \bar{x}, t_{j}\right)<$ $\xi(t, x, s)$ for $t<s<-N_{j}$ and we therefore have

$$
\xi\left(-\infty, \bar{x}, t_{j}\right)<\xi(-\infty, x, s) \text { for } s<-N_{j}
$$

For each $s<-N_{j}$, there exists a $t_{\ell_{j}}<s$ with $\xi\left(t_{\ell_{j}}, x, s\right)<\bar{x}=\xi\left(t_{\ell_{j}}, \bar{x}, t_{\ell_{j}}\right)$. From Corollary 2.4, we conclude that $\xi(t, x, s)<\xi\left(t, \bar{x}, t_{\ell_{j}}\right)$ for $t<t_{\ell_{j}}$. As a consequence,

$$
\xi(-\infty, x, s) \leq y_{1}(\bar{x}) .
$$

We deduce that $\lim _{s \rightarrow-\infty} \xi(-\infty, x, s)=y_{1}(\bar{x})$ by letting $s \rightarrow-\infty, t_{j} \rightarrow$ $-\infty, t_{j}>s$ in view of (3.22) and (3.21).

If we apply the same argument to $y_{2}(\bar{x})$, we complete the proof of our assertion that (3.16) holds for almost all points in $[0, L]$.

Let us now suppose that (3.16) holds. From (3.14), we deduce that

$$
\int_{u_{c}(\xi(t, \bar{x}, \bar{t}), t)}^{u_{c}(\bar{x}, \bar{t})} \frac{f^{\prime}(v)}{g(v)} d v=\bar{x}-\xi(t, \bar{x}, \bar{t})
$$

for all $t \leq \bar{t}$. From (2.4), $v(t)=u_{c}\left(\xi_{+}(t, \bar{x}, \bar{t}), t\right)$ for all $t \leq \bar{t}$. From (3.14), (3.12), and (3.16), this implies that $u_{c}(\xi(t, \bar{x}, \bar{t}), t) \rightarrow a_{2 m}$ as $t \rightarrow-\infty$ monotonically. If we now use (3.23) and (3.16), we have

$$
\lim _{\bar{t} \rightarrow-\infty} \int_{a_{2 m}}^{u_{c}(\bar{x}, \bar{t})} \frac{f^{\prime}(v)}{g(v)} d v=\bar{x}-y(\bar{x}) .
$$

The integrand in this expression is of fixed sign and hence the integral is monotone with respect to $u_{c}(\bar{x}, \bar{t})$. Thus, $\lim _{\bar{t} \rightarrow-\infty} u_{c}(\bar{x}, \bar{t})$ exists.

Since (3.16) holds for almost all points in $[0, L]$ and $\lim _{\bar{t} \rightarrow-\infty} u_{c}(\bar{x}, \bar{t})$ exists, we can define a function $\psi(x)$ by the relation

$$
\lim _{t \rightarrow-\infty} u_{c}(x, t)=\psi(x)
$$


It remains to show that $\psi$ defined by (3.24) is a traveling wave solution of (1.1). To this end, we multiply (1.1) by a test function $\eta(x) \in C^{\infty}\left(S^{1}\right)$ and integrate to obtain

$$
\begin{aligned}
0 & =\int_{0}^{L} \int_{T}^{T+\Delta T}\left(\eta u_{t}-f(u) \eta_{x}-g(u) \eta\right) d t d x \\
& =\left.\int_{0}^{L} \eta u\right|_{T} ^{T+\Delta T} d x+\int_{0}^{L} \int_{T}^{T+\Delta T}\left(-f(u) \eta_{x}-g(u) \eta\right) d t d x .
\end{aligned}
$$

Letting $T \rightarrow-\infty$ and using (3.24) in this expression yields

$$
0=\int_{0}^{L}\left(-f(\psi) \eta_{x}-g(\psi) \eta\right) d x
$$

Therefore, $\psi$ is a traveling wave solution of (1.1) if it is admissible. The admissibility will follow from the following lemma.

Lemma 3.8. Let $u_{c}(x, t)$ be an $L^{\infty}$ bounded solution of (1.1) defined for $t \in \mathbb{R}$ and let $f_{0}$ be given in $\left(\mathrm{H}_{1}\right)$. Then there exists a constant $C$ depending only on $\left\|u_{c}\right\|_{L^{\infty}}$ and $g$ such that, if $x, y \in \mathbb{R}, x>y$ and either $u(x+, \bar{t})$ or $u(x-, \bar{t})$ is greater than either $u(y+, \bar{t})$ or $u(y-, \bar{t})$, then, for $-\infty<t<\bar{t}$,

$$
0<u\left(x^{ \pm}, \bar{t}\right)-u\left(y^{ \pm}, \bar{t}\right)<\frac{C(x-y)}{f_{0}\left(1-e^{-C(\bar{t}-t)}\right)} .
$$

Proof. Let $\xi(t)=\xi(t ; x, \bar{t}), \zeta(t)=\xi(t ; y, \bar{t})$, be given maximal or minimal characterestics. Since characteristics cannot intersect, we have $\xi(t)>\zeta(t)$ for all $t<\bar{t}$. There are functions $v(t), v(\bar{t})=u\left(x^{ \pm}, \bar{t}\right), w(t), w(\bar{t})=u\left(y^{ \pm}, \bar{t}\right)$, such that $(\xi, v)$ and $(\zeta, w)$ are solutions of (2.3). By hypothesis, $v(\bar{t})>$ $w(\bar{t})$. From equation $(2.3), d(v-w) / t=\eta(v, w)(v-w)$, where $\eta(v, w)=$ $[g(v)-g(w)] /(v-w)$. Also, for any constant $r>0$, there is a constant $C=C(r)$ such that $|\eta(v, w)| \leq C$ if $|v|,|w| \leq r$. If $\left\|u_{c}\right\|_{L^{\infty}} \leq r$, then we obtain

$$
v(t)-w(t)=(v(\bar{t})-w(\bar{t})) e^{\left.-\int_{t}^{t} \eta(v(s)), w(s)\right) d s} \geq(v(\bar{t})-w(\bar{t})) e^{-C(\bar{t}-t)} .
$$

From $\left(\mathbf{H}_{1}\right)$, we have $d(\xi-\zeta) / d t=f^{\prime}(v)-f^{\prime}(w)=f^{\prime \prime}(\theta)(v-w)$. Using this relation and (3.25), we obtain

$$
\begin{aligned}
0 & <\xi(t)-\zeta(t)=\xi(\bar{t})-\zeta(\bar{t})-\int_{t}^{\bar{t}} f^{\prime \prime}(\theta(s))(v(s)-w(s)) d s \\
& <\xi(\bar{t})-\zeta(\bar{t})-f_{0}(v(\bar{t})-w(\bar{t})) \int_{t}^{\bar{t}} e^{-C(\bar{t}-s)} d s \\
& =\xi(\bar{t})-\zeta(\bar{t})-f_{0}(v(\bar{t})-w(\bar{t}))\left(1-e^{-C(\bar{t}-t)}\right) \frac{1}{C} .
\end{aligned}
$$

As a consequence of this inequality and the definition of $v(\bar{t})$ and $w(\bar{t})$, we have the assertion in the lemma.

[Proof of Theorem 3.7 continued]. We use this lemma to prove that $\psi$ in (3.24) is admissible. We need only to prove that $\psi\left(x^{-}\right)>\psi\left(x^{+}\right)$for any point of discontinuity $x$ of $\psi$. Indeed, if there is an $x_{0}$ of discontinuity of $\psi$ such 
that $\psi\left(x_{0}^{-}\right)<\psi\left(x_{0}^{+}\right)$, then, by taking $x=x_{0}-\delta, y=x_{0}+\delta, \delta>0$, in Lemma 3.8, we obtain

$$
0<u_{c}\left(x_{0}+\delta, \bar{t}\right)-u_{c}\left(x_{0}-\delta, \bar{t}\right) \leq \frac{2 \delta C}{f_{0}\left(1-e^{-C}\right)} .
$$

Letting $\bar{t} \rightarrow-\infty$ and $\delta \rightarrow 0$ in this relation, we obtain $0 \leq \psi\left(x_{0}^{+}\right)-\psi\left(x_{0}^{-}\right) \leq 0$, which is a contradiction. Thus, $\psi(x)$ is an admissible traveling wave solution of (1.1).

It remains to prove that the oscillation number of $\varphi-a_{2 m}$ is no more than that of $\psi-a_{2 m}$. Recall that

$$
\mathscr{N}_{\varphi-a_{2 m}}=\left\{x \in[0, L]: \varphi\left(x^{+}\right)=\varphi\left(x^{-}\right)=0\right\}
$$

and that

$$
\lim _{t \rightarrow \infty} u_{c}(x, t)=\varphi(x) \quad \text { a.e. }
$$

We claim that

$$
\lim _{t \rightarrow \infty} u_{c}\left(x_{0}, t\right)=a_{2 m} \text { for all } x_{0} \in \mathscr{N}_{\varphi-a_{2 m}} .
$$

If this is not true, then there is a subsequence $\left\{t_{j}\right\}, t_{j} \rightarrow \infty$ as $j \rightarrow \infty$, and an $\epsilon_{0}>0$ such that $\left|u_{c}\left(x_{0}, t\right)-a_{2 m}\right| \geq \epsilon_{0}$ for $j=1,2, \ldots$. Without loss of generality, we can assume that

$$
u_{c}\left(x_{0}, t\right)-a_{2 m} \geq \epsilon_{0}, \quad j=1,2, \ldots,
$$

since the other case can be handled in a similar way. Since $x_{0}$ is a point of continuity of $\varphi$ and (3.27) is satisfied, for any $\delta>0$, there exists an $\bar{x} \in$ $\left(x_{0}-\delta, x_{0}\right)$ such that

$$
\lim _{t \rightarrow \infty} u_{c}(\bar{x}, t)=\varphi(\bar{x}) \leq a_{2 m}+\frac{\epsilon_{0}}{2} .
$$

If we use Lemma 2.8 with $x=x_{0}, y=\bar{x}, \bar{t}=t_{j}, t=0$, we have $0<$ $u_{c}\left(x_{0}, t_{j}\right)-u_{c}\left(\bar{x}, t_{j}\right)<C_{1} \delta$ for some constant $C_{1}$ independent of $j$. If $j \rightarrow$ $\infty$, we obtain $0 \leq \epsilon_{0} / 2 \leq C_{1} \delta$ for all $\delta>0$. This is a contradiction and shows that (3.28) is satisfied.

We now want to show that $\mathscr{N}_{\varphi-a_{2 m}} \subset \mathscr{N}_{\psi-a_{2 m}}$ which implies Card $\mathscr{N}_{\varphi-a_{2 m}}$ $\leq$ Card $\mathscr{N}_{\psi-a_{2 m}}$ and will complete the proof of Part (ii) of Theorem 3.7. Let $x_{0} \in \mathscr{N}_{\varphi-a_{2 m}}$ and let $\xi\left(t ; x_{0}, s\right)$ be $\xi^{ \pm}\left(t ; x_{0}, s\right)$. Then the functions $\xi\left(t ; x_{0}, s\right)$ and $v(t ; s)$, for $t<s$ satisfy the equations

$$
\begin{aligned}
& \frac{d \xi}{d t}=f^{\prime}(v), \quad \frac{d v}{d t}=g(v), \\
& \xi\left(s ; x_{0}, s\right)=x_{0}, \quad v(t ; s)=u_{c}\left(\xi\left(t ; x_{0}, s\right), t\right), \quad v(s ; s)=u_{c}\left(x_{0}, s\right) .
\end{aligned}
$$

Thanks to (3.12), hypothesis $\left(\mathrm{H}_{2}\right)$ and (3.29), if $u_{c}\left(x_{0}, s\right) \neq a_{2 m}$, then we have either

$$
v(s ; s)=u_{c}\left(x_{0}, s\right)<v(t ; s)<a_{2 m}
$$

or

$$
a_{2 m}<v(t ; s)<u_{c}\left(x_{0}, s\right)=v(s ; s) .
$$


If $v=a_{2 m}+\bar{v}$ in (3.29), then an elementary estimate using the variation of constants formula and Gronwall's inequality shows that

$$
|\bar{v}(t ; s)|=\left|v(t ; s)-a_{2 m}\right| \leq C_{2}\left|u_{c}\left(x_{0}, s\right)-a_{2 m}\right| e^{g^{\prime}\left(a_{2 m}\right)(t-s) / 2}
$$

for $t \leq s, s \geq s_{0}$, where $s_{0}, C_{2}$ are constants. Since $g^{\prime}\left(a_{2 m}\right)>0$, we have $v(t ; s) \rightarrow a_{2 m}$ exponentially as $s \rightarrow \infty$. Using (3.29) and (3.31), we obtain, for $t \leq s$,

$$
\begin{aligned}
\left|\xi\left(t ; x_{0}, s\right)-x_{0}\right| & \left.=\mid \int_{s}^{t} f^{\prime}(v(\tau ; s), s)\right) d \tau \mid \\
& =\left|\int_{t}^{s} f^{\prime \prime}(\theta(\tau ; s))\left[v(\tau ; s)-a_{2 m}\right] d \tau\right| \\
& \leq C_{3}\left|u_{c}\left(x_{0}, s\right)-a_{2 m}\right| .
\end{aligned}
$$

From (3.28), this implies that $\lim _{s \rightarrow \infty} \xi\left(t: x_{0}, s\right)=x_{0}$ for all $t \in \mathbb{R}$. Hence, either

$$
u_{c}\left(x_{0}^{+}, t\right)=\lim _{s \rightarrow \infty} u_{c}\left(\xi\left(t: x_{0}, s\right), t\right)=\lim _{s \rightarrow \infty} v(t ; s)=a_{2 m}
$$

or

$$
u_{c}\left(x_{0}^{-}, t\right)=\lim _{s \rightarrow \infty} u_{c}\left(\xi\left(t: x_{0}, s\right), t\right)=\lim _{s \rightarrow \infty} v(t ; s)=a_{2 m}
$$

for all $t \in \mathbb{R}$. From (3.24), we deduce that

$$
\text { either } \psi\left(x_{0}^{+}\right)=a_{2 m} \text { or } \psi\left(x_{0}^{-}\right)=a_{2 m} \text {. }
$$

Since $\psi(x)$ is a traveling wave solution of $(1.1)$ with wave speed $s=f^{\prime}\left(a_{2 m}\right)=$ 0 , we have

$$
0=s=\frac{f\left(\psi\left(x_{0}^{+}\right)\right)-f\left(\psi\left(x_{0}^{-}\right)\right)}{\psi\left(x_{0}^{+}\right)-\psi\left(x_{0}^{-}\right)} .
$$

This relationship, the strict convexity of $f$ and (3.33) imply that $\psi\left(x_{0}^{+}\right)=$ $\psi\left(x_{0}^{-}\right)=a_{2 m}$ and $x_{0} \in \mathscr{N}_{\psi-a_{2 m}}$. Thus, $\mathscr{N}_{\varphi-a_{2 m}} \subset \mathscr{N}_{\psi-a_{2 m}}$. This completes the proof of part (ii) of Theorem 3.7.

Let us now prove part (iii) of the theorem. Suppose that $u_{c}(x, t)$ connects to a constant $b \in\left\{a_{1}, a_{2}, \ldots, a_{2 k+1}\right\}$ at $t=+\infty$. If $b=a_{2 m}$, then the arguments from (3.26) to (3.32) show in effect that $u_{c}(x, t)=a_{2 m}$.

Therefore, suppose that $b=a_{2 m+1}$. Without loss of generality, we may assume that $u_{c}(x, t) \not \equiv a_{2 m+1}$. In view of Lemmas 3.1 and $3.2, \mathscr{R}(u(\cdot, t)) \subset$ $\left(a_{2 m}, a_{2 m+2}\right)$ for all $t \in \mathbb{R}$. We claim that

$$
\begin{gathered}
\text { either } \mathscr{R}\left(u_{c}(\cdot, t)\right) \subset\left(a_{2 m+1}, a_{2 m+2}\right), \\
\text { or } \mathscr{R}\left(u_{c}(\cdot, t)\right) \subset\left(a_{2 m}, a_{2 m+1}\right)
\end{gathered}
$$

for $t \in \mathbb{R}$. If we assume that this is not the case, then the fact that $\mathscr{R}(u(\cdot, t))$ is a closed interval implies that there exist $t_{0}, x_{1}, x_{2} \in \mathbb{R}$ such that

$$
\begin{aligned}
& \text { either } u_{c}\left(x_{1}^{+}, t_{0}\right) \text { or } u_{c}\left(x_{1}^{-}, t_{0}\right) \in\left(a_{2 m}, a_{2 m+1}\right) \cup\left(a_{2 m+1}, a_{2 m+2}\right) \text { and } \\
& \text { either } u_{c}\left(x_{2}^{+}, t_{0}\right) \text { or } u_{c}\left(x_{2}^{-}, t_{0}\right)=a_{2 m+1} .
\end{aligned}
$$

For definiteness, we suppose that

$$
u_{c}\left(x_{1}^{-}, t_{0}\right) \in\left(a_{2 m}, a_{2 m+1}\right), \quad u_{c}\left(x_{2}^{-}, t_{0}\right)=a_{2 m+1} .
$$


The other cases may be discussed in a similar way. Consider the genuine backward characteristics $\xi_{2}(t)=x_{2}$ and $\xi_{1}(t)=\xi\left(t, x_{1}, t_{0}\right)$, which satisfy the equations

$$
\begin{aligned}
& \frac{d \xi_{1}}{d t}=f^{\prime}\left(v_{1}\right), \quad \frac{d v_{1}}{d t}=g\left(v_{1}\right), \\
& v_{1}\left(t_{0}\right)=u_{c}\left(x_{1}^{-}, t_{0}\right), \quad \xi_{1}\left(t_{0}\right)=x_{1} .
\end{aligned}
$$

Therefore, $v_{1}(t) \rightarrow a_{2 m}$ exponentially as $t \rightarrow-\infty$ and $d \xi / d t \rightarrow f^{\prime}\left(a_{2 m}\right)$ as $t \rightarrow-\infty$. This implies that there exists $t<t_{0}$ such that $\xi_{1}$ intersects either $\xi_{2}$ or $\xi_{2}+L$ at $t$, which contradicts Corollary 2.4. This proves (3.34).

Once (3.34) is proved, we can apply the proofs of part (ii) starting from (3.12) to yield that $u_{c}(x, t)-\psi\left(x-f^{\prime}\left(a_{2 m}\right) t\right) \rightarrow 0$ a.e. as $t \rightarrow-\infty$ in case (3.34b) holds and $u_{c}(x, t)-\psi\left(x-f^{\prime}\left(a_{2 m+2}\right) t\right) \rightarrow 0$ a.e. as $t \rightarrow-\infty$ in case (3.34a) holds, where $\psi$ is a traveling wave solution of (1.1) with wave speed $f^{\prime}\left(a_{2 m}\right)$ and $f^{\prime}\left(a_{2 m+2}\right)$, respectively.

\section{THE LIMIT OF PARABOLIC REgULARIZATIONS}

In this section, we consider the parabolic regularization of (1.1),

$$
u_{t}+f(u)_{x}=g(u)+\epsilon u_{x x}, \quad t>0, \quad x \in S^{1},
$$

where $\epsilon>0$ is a parameter. For a fixed time interval $[0, T]$, if $u^{\epsilon}\left(\cdot, t, u_{0}\right)$ is the solution of $(4.1)$ with $u\left(\cdot, 0, u_{0}\right)=u_{0} \mathrm{~L}$. Tartar (1979) has shown that, as $\epsilon \rightarrow 0, u^{\epsilon}\left(\cdot, t, u_{0}\right)$ converges on $[0, T]$ to the solution of $(1.1)$ with the same initial value. For (4.1), Fiedler and Mallet-Paret (1989) have shown that the attractor $\mathscr{A}_{\epsilon}$ of $(4.1)$ in $H^{2}\left(S^{1}\right)$ consists of equilibrium points, traveling waves, and orbit connections between them. As remarked in the introduction, the ultimate goal would be to prove that the limit of $\mathscr{A}_{\epsilon}$ as $\epsilon \rightarrow 0$ is equal to $\mathscr{A}_{0}$. At this time, we can show only that a connecting orbit of $(4.1)$ converges almost everywhere to that of $(1.1)$ as $\epsilon \rightarrow 0$. A similar statement holds also for the traveling wave solutions of (4.1) and (1.1). The method of proof is similar to Tartar (1979).

Theorem 4.1. If $B=\left\{u_{c}^{\epsilon}(x, t), 0<\epsilon \leq \epsilon_{0}\right\}$ is a set of connecting orbits of (4.1), then there is a subsequence $\left\{u_{c}^{\epsilon_{n}}(x, t)\right\}$ of $B$ converging to $u_{c}(x, t)$ as $\epsilon \rightarrow 0$ a.e. in $S^{1} \times \mathbb{R}$ where $u_{c}(x, t)$ is a connecting orbit of (1.1).

Proof. In this proof, we let $u^{\epsilon}=u_{c}^{\epsilon} \in B$. By condition $\left(\mathrm{H}_{2}\right)$ and the maximum principal, the set $B$ satisfies $\max _{u^{\epsilon} \in B}\left\|u^{\epsilon}\right\|_{L^{\infty}} \leq C$. Thus, there is a subsequence of $B$, denoted by $\left\{u^{\epsilon}(x, t)\right\}$ again, and a function $u \in L^{\infty}\left(S^{1} \times \mathbb{R}, \mathbb{R}\right)$ such that

$$
u^{\epsilon} \stackrel{w *}{\rightarrow} u(x, t) \quad \text { in } L^{\infty}\left(S^{1} \times \mathbb{R} ; \mathbb{R}\right) .
$$

Then there is a family of Borel probability measures $\left\{\nu_{(x, t)} ;(x, t) \in S^{1} \times \mathbb{R}\right\}$ such that, for each $g \in C(\mathbb{R})$, we have

$$
g\left(u^{\epsilon}(x, t)\right) \stackrel{w *}{\rightarrow} \bar{g}(x, t) \equiv \int_{\mathbb{R}} g(\lambda) d \nu_{(x, t)}(\lambda) \quad \text { in } L^{\infty}\left(S^{1} \times \mathbb{R}, \mathbb{R}\right) .
$$

Let $\phi \in C^{2}(\mathbb{R})$ be any convex function, let $f$ be as in (4.1) and define

$$
\psi(u)=\int^{u} \phi^{\prime}(s) f^{\prime}(s) d s .
$$


Then

$$
\begin{aligned}
\phi\left(u^{\epsilon}(x, t)\right) \stackrel{w *}{\rightarrow} \bar{\phi}(x, t) & \equiv \int_{\mathbb{R}} \phi(\lambda) d \nu_{(x, t)}(\lambda), \\
\psi\left(u^{\epsilon}(x, t)\right) \stackrel{w *}{\rightarrow} \bar{\psi}(x, t) & \equiv \int_{\mathbb{R}} \psi(\lambda) d \nu_{(x, t)}(\lambda),
\end{aligned}
$$

in $L^{\infty}\left(S^{1} \times \mathbb{R}_{+} ; \mathbb{R}\right)$. By using (4.4) and (4.2), we find that

$$
\phi\left(u^{\epsilon}\right)_{t}+\psi\left(u^{\epsilon}\right)_{x}=\epsilon \phi\left(u^{\epsilon}\right)_{x x}-\epsilon \phi^{\prime \prime}\left(u^{\epsilon}\right)\left(u_{x}^{\epsilon}\right)^{2}+\phi^{\prime}\left(u^{\epsilon}\right) g\left(u^{\epsilon}\right) .
$$

Letting $\phi(u)=u^{2}$ in (4.6) and integrating over $S^{1} \times[0, T]$, we obtain

$$
\begin{aligned}
\int_{S^{1}}\left(u^{\epsilon}(x, T)\right)^{2} d x= & \int_{S^{1}}\left(u^{\epsilon}(x, 0)\right)^{2} d x-\int_{0}^{T} \int_{S^{1}} \epsilon\left(u_{x}^{\epsilon}\right)^{2} d x d t \\
& +2 \int_{0}^{T} \int_{S^{1}} u^{\epsilon} g\left(u^{\epsilon}\right) d x d t
\end{aligned}
$$

It is not difficult to show that $u^{\epsilon}$ is uniformly bounded in $L^{\infty}\left(S^{1} \times \mathbb{R}\right)$. As a consequence, we see from (4.7) that

$$
\sup _{\epsilon>0} \int_{0}^{T} \int_{S^{1}} \epsilon\left(u_{x}^{\epsilon}\right)^{2} d x d t<\infty .
$$

Thus, $\epsilon \phi\left(u^{\epsilon}\right)_{x} \rightarrow 0$ in $L^{2}\left(S^{1} \times[0, T]\right)$ and, hence, $\epsilon \phi(u)_{x x} \rightarrow 0$ in $W^{-1,2}\left(S^{1} \times \mathbb{R}_{+}\right)$. Furthermore, $\epsilon \phi^{\prime \prime}\left(u^{\epsilon}\right)\left(u_{x}^{\epsilon}\right)^{2}$ and $u^{\epsilon} \phi\left(u^{\epsilon}\right)$ are bounded in $\mathscr{M}\left(S^{1} \times[0, T]\right)$, the space of signed Radon measures on $S^{1} \times[0, T]$ with finite mass. As a consequence, the right hand side of (4.6) is compact in $W^{-1,2}\left(S^{1} \times[0, T]\right)$ by Corollary 1 of Chapter 1 of Evans (1990). This enables us to apply the Div-Curl Lemma (Murat(1978)).

Lemma 4.2 (Div-Curl Lemma). Assume that $\left\{v_{k}\right\},\left\{w_{k}\right\}$ are two bounded sequences in $L^{2}\left(U ; \mathbb{R}^{n}\right), U \subset \mathbb{R}^{n}$, such that

(i) $\left\{\right.$ div $\left.v_{k}\right\}$ is compact in $W^{-1,2}(U)$,

(ii) $\left\{\right.$ curl $\left.w_{k}\right\}$ is compact in $W^{-1,2}\left(U ; \mathbb{R}^{n \times n}\right)$.

If $v_{k} \rightarrow v, w_{k} \rightarrow w$ in $L^{2}\left(U, \mathbb{R}^{n}\right)$, then $v_{k} \cdot w_{k} \rightarrow v \cdot w$ in the sense of distributions.

Proof of Theorem 4.1 continued. If we let

$$
v_{\epsilon}=\left(f\left(u^{\epsilon}\right), u^{\epsilon}\right), w_{\epsilon}=\left(\phi\left(u^{\epsilon}\right),-\psi\left(u^{\epsilon}\right)\right)
$$

then

$$
v_{\epsilon} \cdot w_{\epsilon} \stackrel{w *}{\rightarrow} \int_{\mathbb{R}}[f(\lambda) \phi(\lambda)-\lambda \psi(\lambda)] d \nu_{x t}(\lambda)
$$

By Div-Curl Lemma, we also have

$$
v_{\epsilon} \cdot w_{\epsilon} \stackrel{w *}{\rightarrow} \bar{v} \cdot \bar{w}=(\bar{f}, u) \cdot(\bar{\phi},-\bar{\psi}) .
$$

Thus

$$
\int_{\mathbb{R}}[f(\lambda) \phi(\lambda)-\lambda \psi(\lambda)] d \nu(\lambda)=\bar{f}(x, t) \int_{\mathbb{R}} \phi(\lambda) d \nu(\lambda)-u(x, t) \int_{\mathbb{R}} \psi(\lambda) d \nu_{(x, t)}(\lambda)
$$

or

$$
\int_{\mathbb{R}}[(f(\lambda)-\bar{f}(x, t)) \phi(\lambda)+(u(x, t)-\lambda) \psi(\lambda)] d \nu_{(x, t)}(\lambda)=0
$$


If $\phi(\lambda)=|\lambda-u(x, t)|$, then

$$
\psi(\lambda)=\int^{\lambda} f^{\prime}(s) \phi^{\prime}(s) d s=\operatorname{sign}(\lambda-\bar{u}(x, t))(f(\lambda)-f(\bar{u}(x, t))) .
$$

With this choice of $\phi$ and $\psi,(4.10)$ reduces to

$$
0=(f(\bar{u}(x, t))-\bar{f}(x, t)) \int_{\mathbb{R}}|\lambda-u(x, t)| d \nu_{(x, t)}(\lambda)
$$

which implies either $\operatorname{supp} \nu_{(x, t)}=\{u(x, t)\}$ or $f(u(x, t))=\bar{f}(x, t)$. Either case implies that $f(u(x, t))=\bar{f}(x, t)$. Now, if we take $\phi(\lambda)=f(\lambda)-$ $f(u(x, t))$ and hence $\psi(\lambda)=\int^{\lambda} f^{\prime 2}(s) d s$, then (4.10) yields

$$
\int_{\mathbb{R}}\left[(f(\lambda)-f(u))^{2}-(\lambda-u) \int_{\tilde{u}}^{\lambda} f^{\prime 2}(s) d s\right] d \nu_{(x, t)}(\lambda)=0 .
$$

We know that the integrand in (3.19) satisfies

$$
\left(\int_{\bar{u}}^{\lambda} f^{\prime}(s) d s\right)^{2}-(\lambda-\bar{u}) \int_{\bar{u}}^{\lambda} f^{\prime 2}(s) d s \leq 0
$$

by Hölder's inequality, where the equality holds only if $f^{\prime}=$ constant or $\lambda=\bar{u}$. In our case, $f^{\prime \prime} \neq 0$ and hence the integrand $<0$ unless $\lambda=u$. Thus (4.11) implies that supp $\nu_{(x, t)}=\{u(x, t)\}$. This proves the pointwise convergence of $\left\{u^{\epsilon}\right\}$ to $u(x, t)$ on $S^{1} \times(-T, T)$. By a diagonalization process, we can further extract a subsequence, denoted by $\left\{u^{\epsilon}\right\}$ again, such that $u^{\epsilon}(x, t) \rightarrow$ $u(x, t)$ a.e. in $S^{1} \times \mathbb{R}$. It is clear that $u(x, t)$ is a solution of $(1.1)$ defined for all $t \in \mathbb{R}$ and hence, by Theorem 3.8, $u(x, t)$ is a connecting orbit of (1.1).

Theorem 4.3. Let $\left\{\varphi^{\epsilon}\left(x-c_{\epsilon} t\right), 0<\epsilon \leq \epsilon_{0}\right\}$ be any sequence of traveling wave solutions of (4.1). Then there is a subsequence of $\left\{\varphi^{\epsilon}\left(x-c_{\epsilon} t\right\}\right.$, denoted by $\left\{\varphi^{\epsilon}\left(x-c_{\epsilon} t\right)\right\}$ again, such that

$$
\varphi^{\epsilon}\left(x-c_{\epsilon} t\right)-\varphi(x-c t) \rightarrow 0
$$

a.e. in $S^{1} \times \mathbb{R}$ as $\epsilon \rightarrow 0$, where $\varphi$ is a traveling wave solution of (1.1).

Proof. Applying Theorem 4.1, we know that

$$
\varphi^{\epsilon}\left(x-c_{\epsilon} t\right) \rightarrow \varphi(x, t) .
$$

a.e. in $S^{1} \times \mathbb{R}$ as $\epsilon \rightarrow 0^{+}$. It remains to prove that $\varphi(x, t)$ is a traveling wave solution of (1.1), extracting a further subsequence of $\left\{\varphi^{\epsilon}\right\}$ if necessary. Indeed, we can assume that $c_{\epsilon} \rightarrow c$ as $\epsilon \rightarrow 0^{+}$without loss of generality. If $\xi=x-c t, t=t$, then

$$
-c \varphi_{\xi}^{\epsilon}+f\left(\varphi^{\epsilon}\right)_{\xi}-g\left(\varphi^{\epsilon}\right)=\left(c_{\epsilon}-c\right) \varphi_{\xi}^{\epsilon}+\epsilon \varphi_{\xi \xi}^{\epsilon} .
$$

By applying (4.12), we can see that $-c \varphi_{\xi}+f(\varphi)_{\xi}-g(\varphi)=0$ in the sense of distributions; that is, $\varphi$ is a traveling wave solution of $(1.1)$ with speed $c$. 


\section{REFERENCES}

A. V. Babin and M. I. Vishik (1989), Attractors of evolutionary equations, "Nauka", Moscow; English transl., North-Holland, Amsterdam, 1992.

C. M. Dafermos (1977), Generalized characteristics and the structure of solutions of hyperbolic conservation laws, Indiana Univ. Math. J. 26, 1097-1119.

L. Evans (1990), Weak convergence methods for nonlinear partial differential equations, CBMS Regional Conf. Ser. in Math., vol. 74, Amer. Math. Soc., Providence, RI.

H. Fan and J. K. Hale (1992), Large time behavior in inhomogeneous conservation laws, Arch. Rat. Mech. Anal. (to appear).

B. Fiedler and J. Mallet-Paret (1989), A Poincaré-Bendixson theorem for scalar reaction diffusion equations, Arch. Rational. Mech. Anal. 107, 325-345.

A. F. Filippov (1960), Differential equations with discontinuous right hand side, Mat. Sb. (N.S.) 42, 99-128; English transl., Amer. Math. Soc. Transl. Ser. 2, Amer. Math. Soc., Providence, RI.

J. K. Hale (1988), Asymptotic behavior of dissipative systems, Amer. Math. Soc., Providence, RI.

S. N. Kruzkov (1970), First order quasilinear equations in several independent variables, Mat. Sb. (N.S.) 81, 228-255; English transl., Math. USSR-Sb. 10, 217-243.

A. N. Lyberopoulos (1992), Large time structure of solutions of scalar conservation laws with a nonlinear source field, preprint.

F. Murat (1978), Compacité par compensation, Ann. Scuola Norm. Sup. Pisa Cl. Sci. (4) 5, 485-507.

L. Tartar (1979), Compensated compactness and applications to partial differential equations, Heriot-Watt Sympos. in Vol. IV, Pitman, New York.

R. Temam (1988), Infinite dimensional dynamical systems in mechanics and physics, Appl. Math. Sci., no. 68, Springer-Verlag, Berlin and New York.

A. I. Vol'pert (1967), The space BV and quasilinear equations, Mat. Sb. (N.S.) 73; English transl., Math. USSR-Sb. 2, 225-267.

Center for Dynamical Systems and Nonlinear Studies, Georgia Institute of TechNOLOGY, ATlanta, GeORgia 30332

E-mail address: hale@math.gatech.edu

Current address (Haitao Fan): Department of Mathematics, Georgetown University, Washington, DC 20057

E-mail address: fan@gumath1.math.georgetown.edu 